\title{
Optical Polarization and Line Variability in Quasars with Associated Absorption
}

\section{T. Aldcroft}

Smithsonian Astrophysical Observatory, Cambridge MA 02138

J. Bechtold, P. Smith, C. Foltz, G. Schmidt

Steward Observatory, Univ. of Arizona, Tucson AZ 85721

\begin{abstract}
The physical origin of associated absorption lines $\left(z_{a b s} \approx\right.$ $\left.z_{q s o}\right)$ which are observed in radio-loud quasar is generally not well understood. Two observable properties, line variability and quasar optical polarization, can serve to constrain the possibilities. To this end, we have obtained high-resolution spectra $(1 \AA)$ and broad-band optical polarizations for over 30 radio-loud quasars with known CIV associated absorption. The quasars are at redshifts between about 1 and 2 , and were previously observed spectroscopically at similar resolution between 1985 - 1986 by Foltz et al (1987). The associated CIV absorption systems include a variety of velocity profiles from weak single lines to very complex multi-component systems to the BAL-like profile of PHL $1157+0128$. In this poster we show the first strong evidence for line variability in $z>1$ radio-loud quasars. We find that in general the variability is less than $\sim 30 \%$. The optical polarization of CIV absorbed quasars is consistent with all radio-loud quasars.
\end{abstract}

\section{Sample and Observations}

The sample of quasars in this study were selected by Foltz et al. (1987) from Veron-Cetty and Veron catalog by: Dec $>-20^{\circ}, \mathrm{S}(11 \mathrm{~cm})>100 \mathrm{mJy}, V<18.5$, and $1.25 \leq z_{e m} \leq 2.4$. The quasars span 4 magnitudes in absolute luminosity and radio spectral indices covering $-1.2 \lesssim \alpha \lesssim 0.5\left(S_{\nu} \propto \nu^{\alpha}\right)$. Foltz obtained spectra ( $1 \AA$ resolution) covering the CIV emission peak for 90 of these quasars, using the MMT during 1985 - 1986.

47 of the 90 quasars were identified as having associated CIV absorption. We obtained broadband optical polarizations for 40 of the 47 at the Steward 61 " telescope in 1995 and 1996 . In most cases the $1-\sigma$ uncertainty was 0.5 $0.7 \%$. We obtained spectra ( $1 \AA$ resolution) for 31 of the 47 absorbed quasars during 1995 - 1996 using the MMT. In most cases the S/N of the new spectrum is comparable to, or better than, the old spectrum. 


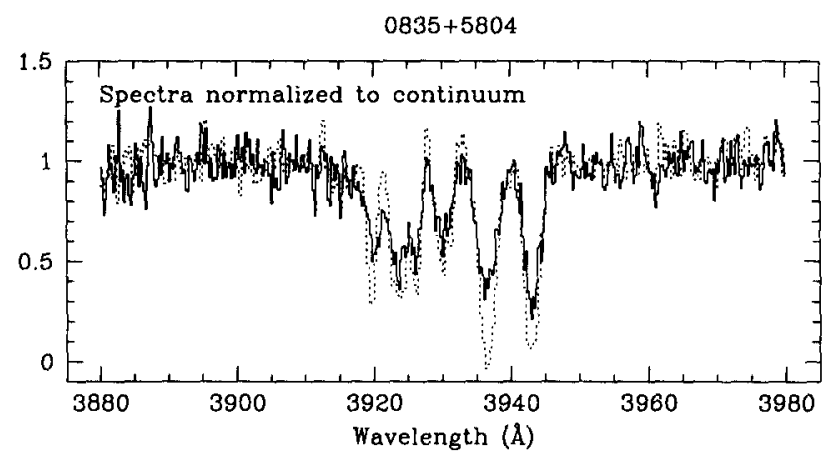

Figure 1. Overlay of spectra from 1985 (solid) and 1995 (dotted) for Q0835+5804 (3C 205)

\section{Results}

In the $40 \mathrm{CIV}$ absorbed quasars with measured optical polarization, we found only one "high polarization quasar", with polarization above $3 \%$. This is consistent with the rate for quasars selected without regard for associated absorption. Therefore we find no correlation of CIV absorption and high polarization. This contrasts with the trend for $\mathrm{Mg}$ II absorbed quasars (Aldcroft, in prep).

We analyzed the variability of the ensemble of associated absorption lines, using non-associated lines (which do not vary) as a control. We found that associated line variability on time scales of 3-5 years (quasar frame) is less than $\sim 30 \%$. In narrow associated lines in radio-quiet QSOs, variability by factors of 3-4 has been observed (Hamann et al. 1994, Barlow et al. 1996)

In two individual cases (3C 205 and PHL 1157+014), we found strong evidence for variability at a level of $\sim 20 \%$. The spectra from 1985 and 1995 for $3 \mathrm{C} 205$ are overlayed in Figure 1. The doublet on the right side is well-resolved and has clearly strengthened. These are the first examples of associated absorption line variability in radio-loud quasars at $z>1$. This is indicates that the material causing absorption in these cases is physically associated with the quasar.

\section{References}

Barlow, T., et al., 1996, preprint

Foltz, C., et al., 1988, in QSO Absorption Lines, eds. J. C. Blades, D. A. Turnshek, \& C. A. Norman (Cambridge: Cambridge Univ. Press)

Hamann, F., et al., 1994, BAAS, 184, \#11.07 